\title{
Fluoroquinolones Reported Hepatotoxicity
}

\author{
Elias Adikwu*, Oputiri Deo
}

Department of Pharmacology, Faculty of Basic Medical Sciences, College of Health Sciences, University of Port Harcourt, Port Harcourt, Nigeria.

Email: *adikwuelias@gmail.com

Received March $4^{\text {th }}, 2012$; revised April 10 $0^{\text {th }}, 2012$; accepted May $4^{\text {th }}, 2012$

\begin{abstract}
Fluoroquinolones are known to be safe and well tolerated. They are said to have the widest clinical acceptability when compared with other antibiotics. Their reported side effects include gastrointestinal tract, central nervous system effect and blood disorder. Rare side effects include phototoxicity, hypersensitivity, convulsion, psychosis, tendinitis, hypoglycemia, cardiotoxicity and nephrotoxicity. Some of these side effects have led to the withdrawal of some fluoroquinolones like travofloxacin from clinical use in some countries. Of recent fluoroquinolones induce cardiotoxicity and hepatotoxicity has gain attention. Due to increasing reports on fluoroquinolones associated hepatotoxicity in experimental Animal studies and clinical experience. This study reviews reported hepatotoxicity associated with clinically used fluoroquinolones and their safety profile on liver function. It was observed that some fluoroquinolones may have hepatotoxic potential. Reported fluoroquinolones induce hepatotoxicity manifested as hepatitis, pancreatis, jaundice, liver injury and hepatic failure. Most reported cases of fluoroquinolones induced hepatotoxicity were marked by elevated levels of alanine aminotransferase, aspartate aminotransferase, alkaline phosphatase, direct bilirubin, total bilirubin and prolong prothrobin time. In some reported cases liver biopsy revealed hepatocellular damage, necrosis and degeneration. Mixed inflammatory infiltrates containing eosinophils, portal edema, bile ductular proliferation and lobular cholestasis were also observed in some cases. The mechanism of fluoroquinolones induce hepatotoxicity may involve generation of oxidative radicals in the liver during drug metabolism which induces DNA damage, mitochondrial damage and gene regulation leading to hepatocellular damage. This was observed in travofloxacin which enhances hepatic mitochondrial peroxynitrite stress in mice with underlying increased basal levels of super oxide leading to the disruption of critical mitochondrial enzyme and gene regulation. This mechanism could be associated with fluoroquinolones mechanism of action which includes DNA damage. In conclusion fluoroquinolones are well tolerated but some may have hepatotoxic potential. Most clinically used fluoroquinolones are relatively safe but Clinicians should consider patients liver function status before fluoroquinolones clinical recommendation. In some cases biochemical parameters associated with liver function should be monitored in patients with impaired liver function.
\end{abstract}

Keywords: Fluoroquinolone; Liver; Toxicity

\section{Introduction}

Fluoroquinolones are a series of synthetic antibacterial agents that are used in the treatment of a variety of bacterial infections. The first quinolone, nalidixic acid was introduced in 1962. Since then, structural modifications have resulted in second-, third-, and fourth-generation fluoroquinolones, which have improved coverage of gram-positive organisms [1].

Fluoroquinolones induce their action by inhibiting DNA synthesis through promoting cleavage of bacterial DNA in the DNA-enzyme complexes of DNA gyrase and type IV topoisomerase, resulting in rapid bacterial death [2]. The fluoroquinolones as a class are generally well tolerated most adverse effects are mild $[3,4]$. The

${ }^{*}$ Corresponding author. most common adverse events associated with the quinolone class involve the gastrointestinal tract (nausea and diarrhea) and central nervous system (CNS) (headache and dizziness). These adverse events are usually mild and do not require discontinuation of therapy. Uncommon and potentially serious quinolone-related adverse events involve the cardiovascular system (rate-corrected electrocardiographic QT interval prolongation), musculoskeletal system (tendinitis and tendon rupture), endocrine system (glucose homeostasis dysregulation), renal system (crystalluria, interstitial nephritis, and acute renal failure), and the CNS (seizures) [5].

Severe idiosyncratic adverse events are specific to individual agents that may share some structural congruity, such as the 1-(2,4)-difluorophenyl group shared by travofloxacin (associated with hepatitis), temafloxacin (as- 
sociated with hemolytic-uremic syndrome), and tosufloxacin (associated with eosinophilic pneumonitis). Overall fluoroquinolones with higher discontinuation rates, such as travofloxacin and grepafloxacin, are no longer available for general use [6].

Recently there are reported cases of fluoroquinolones induced hepatotoxicity, Ciprofloxacin [7], Moxifloxacin [8], Gatifloxacin [9], Norfloxacin [10], Ofloxacin [11] and Levofloxacin [12]. These were marked by elevated levels of some biochemical parameters (alanine aminotransferase, aspartate aminotransferase, alkaline phosphatase, direct bilirubin, total bilirubin and prolong prothrobin time). Hepatocellular damage, necrosis and degeneration were observed. Hepatotoxic reactions (liver failure, hepatic dysfunction and pancreatitis) have led to travofloxacin being either restricted for use in patients with life or limb threatening infections for which no suitable safe and effective alternatives are available or suspended from use in Europe [13]. This work critically analyzed reported hepatotoxicity induced by fluoroquinolone and their safety on hepatic function.

Gatifloxacin is one of the most frequently prescribed fluoroquinolone. It is known to be safe and well tolerated with very few side effects [14]. Despite its safe pharmacological profile it may have hepatotoxic potential [15]. Cheung et al. (2004) [16] reported a case of gatifloxacin induced hepatotoxicity and acute pancreatitis in a patient administered $400 \mathrm{mg} /$ day. Patient laboratory evaluation revealed elevated levels of aspartate aminotransferase, alanine aminotransferase, alkaline phosphatase and bilirubin. Liver biopsy showed portal edema, bile ductular proliferation and portal inflammatory infiltrate that included eosinophils with lobular cholestasis.

A case of possible fulminant hepatic failure induced by gatifloxacin was also reported by Coleman et al. (2002) [17]. It was characterized by abnormal liver aspartate aminotransferase, alanine aminotransferase and hepatocellular necrosis. The patient liver function steadily declined eventually resulting in multiple organ failure and patient death. Another gatifloxacin associated hepatitis which percutaneous liver biopsy showed acute hepatitis was also reported. Eosinophilic infiltrates consistent with drug induced hepatitis was seen. Despite reported gatifloxacin associated hepatotoxicity, clinical trials and post marketing study established it is safe on the liver. This can be seen from a single well-documented case of reversible gatifloxacin-associated hepatitis that was reported in a post marketing study of 15,625 patients [18]. In the evaluation of the pharmacokinetics profile of gatifloxacin in infants and Children, 118 Pediatric patients were enrolled. 111 patients were doused with gatifloxacin. It was observed that only three (3) subjects experienced transient increased in liver function test which returned to normal without intervention. This probably showed that gatifloxacin may not be hepatotoxic in children [19].

The safety profile of gatifloxacin on hepatocytes can also be seen from fluoroquinolone clinical trials by drug induced liver injury network (DILIN) involving 679 registrants. Only one gatifloxacin induced hepatotoxicity was recorded [20]. The safety of gatifloxacin on liver function was also reported by some authors [21]. In this review it was observed that only few cases of gatifloxacin associated hepatotoxicity were reported. This could be used as yardstick for the safety of this drug on liver function.

Levofloxacin is generally well tolerated and has a good Safety Profile. It is known, to have few adverse effects [22]. Levofloxacin induced hepatotoxicity was reported in humans. Coban et al. (2005) [23] reported a case of levofloxacin induced acute fulminant hepatic failure in a patient with chronic hepatitis B Infection. Laboratory findings revealed elevated levels of total bilirubin, direct bilirubin, alkaline phosphates, alanine aminotransferase, and aspartate aminotransferase. Histopathological examination of the liver revealed ductular proliferation, neutrophil and lymphocytic infiltration in the portal area of the liver. Diffuse hepatocellular necrosis and severe degenerative changes were observed. Numerous apoptotic bodies and intracytoplasmic bile accumulation were seen. Levofloxacin was also reported to induce acute hepatitis in a patient with renal insufficiency and hepatic enzymes were elevated [24,25].

Possible Levofloxacin induced acute hepatocellular injury in a patient with chronic obstructive lung disease was documented by Karim et al. (2001) [26]. Liver function test showed elevated levels of aspartate aminotransferase, alanine aminotransferase and alkaline phosphatase. In a case of 17 individuals with suspected latent multidrug-resistant tuberculosis infection who were treated with pyrazinamide and levofloxacin, 5 cases of hepatocellular injury were reported which may be associated with levofloxacin [27]. This report is an agreement with the observation of Schwalm and Lee (2005) [28] who reported a case of Levofloxacin induced hepatitis marked with elevated liver enzymes. This is further supported by the report of Spahr et al. (2001) [29] who observed acute fatal hepatitis related to levofloxacin. These findings also agree with the report of other scholars who observed severe liver damage induced by Levofloxacin [30].

Furthermore, from Jan 1, 1997 to June 30, 2006 Health Canada received 44 domestic report of liver and biliary disorder associated with levofloxacin. Out of the 44 cases, 5 were hepatic failure, 9 were hepatitis 1 was hepatorenal syndrome while 29 people were associated with elevated liver enzyme levels, cholestatic hepatitis and Jaundice [31]. This is at variance with clinical trials involving 
7000 patients which showed no evidence of serious levofloxacin associated hepatotoxicity. Levofloxacin hepatotoxicity could occur after 6 - 14 days of initiation of therapy. Most reported cases of hepatotoxicity are not associated with hypersensitivity and occurred in patients 65 years of age and above [32].

Despite reported levofloxacin associated hepatotoxicity, reports on the safety profile of Levofloxacin from clinical trials and post Marketing Surveillance showed that it is safe with hepatotoxicity in less than 1 per million people [33]. This report agrees with the information on the safety profile of levofloxacin in Japan. It was reported that levofloxacin induced hepatotoxicity has a very low incidence of 1/100,000 [34]. Incidence of acute hepatotoxicity associated with levofloxacin may be low due to few reported cases from the result of post marketing surveillance. Levofloxacin induce hepatotoxicity is rare but physicians should be aware of possible liver injury that could associated with Levofloxacin.

Ofloxacin is a racemic mixture of levofloxacin and dextrofloxacin. It is well tolerated and is reported to be safe [35]. In this review it was observed that there are only few reported cases of hepatotoxicity associated with ofloxacin. Jones and smith (1997) [36] reported a case of ofloxacin induced jaundice. Laboratory evaluation revealed elevated levels of bilirubin, aspartate aminotransferase and alkaline phosphatase. A similar case of ofloxacin induced severe hepatitis was also documented [37]. The safety profile of ofloxacin on the liver was also established in a sub chronic toxicity study of a fixed dose combination of ofloxacin and ornidazole in musmusculus mice [38]. Despite the safety of ofloxacin on liver function the excretion of ofloxacin may be reduced in patients with severe liver function disorders and a maximum dose of $400 \mathrm{mg}$ of ofloxacin per day should therefore not be exceeded [39]. This review observed that ofloxacin may not be hepatotoxic due to limited reports of impairment. It could be said that ofloxacin is safe on the liver.

Norfloxacin induced hepatotoxicity is very rare and very limited literatures are available [40]. Among the few reported cases of hepatotoxicity associated with norfloxacin include the report of Romero-Gomez et al. (1991) [41] who documented a case of norfloxacin induced cholestatic hepatitis in a patient with alcoholic liver Cirrhosis. Norfloxacin induced cholestatic jaundice with signs of chronic liver failure was reported. It was characterized by elevated levels of aspartate aminotransferase, alanine aminotransferase, total bilirubin, direct bilirubin and alkaline Phosphatase [42]. This report agreed with other observations [43,44]. Bjornson et al. (2000) [45] published a case of norfloxacin induced eosinophilic necrotizing granulomatous hepatitis.

In rats it was observed that administration of norfloxacin induce DNA damage in the liver and fetal tissues after transplacental exposure. Single oral doses of norfloxacin (4, 2, 1, $0.5 \mathrm{MMole} / \mathrm{kg})$ administered to pregnant rats significantly increased the frequency of micro nucleated polychromatic erythrocytes both in fetal and maternal liver and bone marrow [46].

In a clinical trial, 52 healthy subjects and 1980 patients with urinary tract infections or prostatitis received norfloxacin. Among the abnormal laboratory values observed are alanine aminotransferase (1.4\%), aspartate aminotransferase (1.4\%) and alkaline phosphatase (1.1\%). This showed that norfloxacin may not be hepatotoxic, but surveillance reported some cases of hepatic failure [47]. Comparatively norfloxacin could be said to as safe as ofloxacin on the liver due to low incidences of reported cases.

Moxifloxacin is a fluorinated Quinolone that is reported to have a good clinical safely profile [48]. There are few reported cases of hepatic failure due to Moxifloxacin. Clinical experience showed that moxifloxacin may have a minimal hepatotoxic effect. Moxifloxacin induced fatal hepatotoxicity was reported. It was characterized by elevated levels of alkaline phosphatase, alanine aminotransferase, aspartate aminotransferase and total bilirubin. Scan revealed mild splenomegaly [49]. This agreed with the findings of Nori et al. (2004) [50] who documented a case of fulminant hepatic failure characterized by elevated liver enzymes in a patient treated with moxifloxacin. Similar observations were also reported by some researchers [51].

Treatment with moxifloxacin could be associated with a risk of developing fulminant hepatitis potentially leading to life threatening liver failure. Sixteen cases of hepatitis attributed to moxifloxacin were reported to Food and Drug Administration's Adverse Event Reporting System on December 14, 2001 [52].

In a 13 week toxicity evaluation of the safety profile of moxifloxacin in rat, $100 \mathrm{mg}, 500 \mathrm{mg}, 750 \mathrm{mg} / \mathrm{kg}$ of administered moxifloxacin elevated levels of alanine aminotransferase, aspartate aminotransferase, alkaline phosphatase and bilirubin concentration. Scan revealed mild splenomegaly. Administration of these doses for 6 months resulted in increased numbers of degenerative necrotic hepatocytes, scattered diffusely in the liver lobule in most male rats.

Administration of $250 \mathrm{mg} / \mathrm{kg}$ of moxifloxacin to monkeys increased levels of alanine aminotransferase and aspartate aminotransferase [53]. Reported cases of moxifloxacin induced hepatotoxicity are relatively low compared to the number of moxifloxacin prescriptions. In reassuring the safety profile of moxifloxacin on liver function it was reported that it has no significant associated hepatotoxicity [54]. In clinical trials using patients with anti-tuberculosis induced hepatic disease addition of moxifloxacin to anti tuberculosis regimen did not im- 
posed additional hepatotoxicity on the patients [55].

An over view of the cumulative safety data on both oral and intravenous moxifloxacin therapy including data from the most recent clinical trials and post marketing studies showed the safety of moxifloxacin on liver function [56].

Studies showed that moxifloxacin dose adjustment does not appear to be necessary in elderly patients with mild to moderate hepatic function [57,58]. Clinicians should use moxifloxacin with caution in patients with pre-existing liver disease or those at risk of having liver disease.

Ciprofloxacin is said to be generally well tolerated with remarkably few reactions. Ciprofloxacin is not known to be hepatotoxic, but off recent there are more reported cases of ciprofloxacin induce hepatotoxicity [59]. A case of ciprofloxacin induce cholestatic jaundice was reported by Labowitz and Silverman (1997) [60]. Laboratory investigation showed elevated levels of total bilirubin, direct bilirubin, alkaline phosphatase, aspartate aminotransferase and prolong prothrobin time. This report agreed with other findings [61-63]. Ciprofloxacin induced liver injury marked with elevated liver enzymes was also documented. Liver biopsy revealed extensive hepatocellular necrosis involving zones 3 and 2 of hepatic acini and a mixed inflammatory infiltration containing abundant eosinophils [64,65]. Similar findings were also reported by some scholars [66-68]. Ciprofloxacin induced fulminant hepatic failure and hepatitis was also documented [69-72]. Cases of ciprofloxacin induced cholestatic Jaundice were also reported in humans by some authors [73]. Hepatotoxic potential of ciprofloxacin was also seen in animal models. Channa and Janjua (2003) [74] showed that ciprofloxacin administered to rat significantly decreased fetal liver weight, with increase degenerative cells in zone 11 and 111 of the fetus liver [74]. This report was supported by other observations [75, 76].

Furthermore administration of the therapeutic and double therapeutic doses of ciprofloxacin (57 and 114 mg $1 \mathrm{~kg}$ ) in two periods (Pre implantation and post implantation of pregnancy) induced damage in the liver of pregnant rats and fetuses. These changes varied from dilatation of hepatic portal vein, sinusoids, increased Kupffer and inflammatory Cells. Degeneration progressed to necrosis and focal fibrosis. In addition, the liver of the fetuses showed fatty changes, haemolysis of the blood in Sinusoidal Space. Severe dilation of central vein, focal hemosiderosis, necrosis and Pyknotic nuclei were observed [77]. Weyers et al. (2002) [78] reported increased in lipid hydroperoxide (COOH) in the liver of mice exposed to ciprofloxacin. Increased in lipid hydroperoxide $(\mathrm{COOH})$ is a marker of ciprofloxacin induced stress in the liver.
On the contrary other researchers reported the safety of ciprofloxacin on the hepatic system. One of such reports is the ability of Ciprofloxacin to reverse inhibitory effects of ethanol and carbon tetrachloride induced models of hepatic Injury [79]. In this review it was observed that ciprofloxacin may have hepatotoxic potential due to reported cases. Clinicians should take into cognizance liver function status of patients before prescription of ciprofloxacin.

Travofloxacin is a highly known hopatotoxin according to US Food and Drug Administration [80]. More than 100 cases of travofloxacin induced hepatotoxicity were reported. Fourteen of these patents had acute liver failure with transplantation required in four patients, five of the patients died of liver disease. A case of travofloxacin hepatotoxicity was reported which was marked with elevated levels of serum aspartate aminotransferase, alanine amino transferase, alkaline phosphatase, direct bilirubin and total bilirubin. Liver biopsy revealed focal periportal necrosis and eosinophilic infiltration. The sinusoids were dilated and contained lymphocytes and eosinophils [81]. This report agreed with a case of travofloxacin induce hepatotoxicity presented by Lazarczyk et al. (2001) [82] in which liver biopsy revealed extensive centrilobular hepatocytes necrosis which interfered with normal blood flow from the liver. Eosinophils were also observed in liver parenchyma.

Pannu et al. (2001) [83] documented a case of acute liver failure due to travofloxacin which was characterized by extensive liver necrosis and abscess. The right lobe appeared swollen with areas of low attenuation comparable with edema and fatty infiltration. Low attenuation in the left lobe of the liver is compatible with infarction. Angiogram revealed occlusion of the celiac axis with reconstitution of the hepatic artery and cavernous transformation of portal vein. This report has correlation with the findings of Borlak (2004) [84] who reported hepatic injury related to travofloxacin.

In December 1997, 152 cases of serious hepatic events related to travofloxacin were reported to US Food and Drug Administration and 140 cases to the European Agency for Evaluation of medicinal product. A case of travofloxacin induced acute hepatitis was presented with elevated levels of total bilirubin, direct bilirubin, alkaline phosphatase, alanine aminotransferase and inflammatory eosinophilic infiltrates [85]. Travofloxacin was approved in 1997, before its regulatory approval, there were no cases of hepatic failure or death in more than 7000 patients treated with travofloxacin as reported [86].

The April 1998 quarterly report, listed 3 cases of increased liver enzymes associated with eosinophilic infiltration of the liver in patients after less than 14 days of therapy. Among the 11 serious safety reports for liver toxicity as of June 1998 the most typical was a healthy 
female who developed evidence of an eosinophilic hepatitis with no other obvious etiology [87].

Since February 1998, 140 documented cases of serious liver reactions largely in the US have been reported with the use of travofloxacin [88]. During the post marketing period travofloxacin associated liver enzyme abnormalities and symptomatic hepatitis occurred during short term or long term therapy. Liver enzymes abnormalities have been reported in both men and women. Liver failure marked by hepatic necrosis and eosinophilic infiltration have also been reported. Symptomatic pancreatis was also reported on therapy [89].

Animal studies showed that travofloxacin has hepatotoxic potential. Hepatotoxic effect was observed in treated dogs with elevated liver enzymes levels which correlated with centrilobular hepatocellular vacuolar degeneration and necrosis at doses exceeding therapeutic doses [90]. Rats given a non hepatotoxic dose of lipopolysaccharide followed by a non toxic dose of travofloxacin developed significant hepatocellular injury [91]. Shaw et al. (2007) [92] also showed that travofloxacin induced hepatocellular injury in treated mice. In a six month rat toxicological study it was discovered that a dose related increase in the incidence of minimal to mild fatty changes in the liver of male rats treated with travofloxacin occurred. In a six month dog toxicological study hepatocellular vacuolar degeneration and necrosis with elevated liver enzymes were seen. In six month toxicity study involving 16 dogs, there was threefold increase in liver enzymes. Liver biopsy revealed liver damage marked by necrotizing hepatocellular inflammation [93]. Due to travofloxacin induced hepatotoxicity the manufactures withdrew it from European market on 15 June 1999 [94].

The mechanism of fluoroquinolones induced hepatotoxicity is not well understood. Many researchers have given different views. Some researchers stated that the generation of oxidative radicals involved in mitochondrial damage, RNA processing, transcription and inflammation may serve as a mechanism for hepatotoxicity induced by fluoroquinolones [95,96]. This mechanism was supported by Hsiao et al. (2010) [97] who reported that travofloxacin enhances hepatic mitochondrial peroxynitrite stress in mice with underlying increased basal levels of super oxide leading to the disruption of critical mitochondrial enzyme and gene regulation. It was also reported that travofloxacin induced hepatotoxicity may be medicated through the oxidation of the cyclopropylamine substructure to reactive intermediates that may form covalent adducts to hepatic proteins, resulting in damage to liver tissues. This mechanism induced by travofloxacin may be applicable to other fluoroquinolones [98].

Researchers have also shown that one of the mecha- nisms for drug induced hepatotoxicity is through mitochondrial damage as earlier reported. It is known that mitochondrial impairment is usually a final event common to pathways leading to necrotic and apoptotic cell death. Drug induced participation of mitochondria in hepatocellular death is a direct result of drug acting on these organelles [99]. Fluoroquinolones antibacterial activity has been ascribed to DNA binding resulting $\mathrm{n}$ a marked inhibition of bacterial DNA topoisomerase [100]. This mechanism could be further buttress as one of the mechanisms used by fluoroquinolones to alter intracellular cAMP, Calcium levels, suppress DNA, protein Synthesis and mitochondria damage in the acini cells of the liver [101]. One of the factors that could lead to DNA damage and mitochondrial depletion in the liver is probably the ability of fluoroquinolones to generate oxidative radicals in the in the liver as a result of their metabolism by Cytochrome p 450 in the liver as exhibited by Ciprofloxacin [102].

In conclusion most fluoroquinolones are well tolerated and safe, some may have hepatotoxic potentials. In some instances Clinicians should consider patients liver function status before fluoroquinolones clinical recommendation.

\section{REFERENCES}

[1] C. M. Oliphant and G. M. Green, "Quinolones: A Comprehensive Review,” American Family Physician, Vol. 65, No. 3, 2002, pp. 455-464.

[2] P. B. Iannini, R. Kubin, C. Reiter and G. Tillotson, "Reassuring Safety Profile of Moxifloxacin,” Clinical Infectious Disease, Vol. 32, No. 1, 2001, pp. 1112-1114. doi:10.1086/319615

[3] A. J. Schaeffer, "The Expanding role of Fluoroquinolones," American Journal of Medicine, Vol. 113, Suppl. 1A, 2002, pp. 45-54.

[4] H. Haixiao, L. Jun xiang, J. Yidun and L. Linguan, "Severe Liver Damage Caused by Levofloxacin," Adverse Drug Reaction Journal, Vol. 1, 2009, p. 22.

[5] R. C. Owens and P. Ambrose, "Antimicrobial Safety: Focus on Fluoroquinolones," Clinical Infections Disease, Vol. 41, No. 51, 2005, pp. 44-57.

[6] European Agency for the Evaluation of Medicinal Products, "Public Statement on Travofloxacin Alatrofloxacin: Recommendation to Suspend the Marketing Authorization in the European Union,” London, 15 June 1999.

[7] M. K. Bhagirath, "A Case Report of Highly Suspected Ciprofloxacin-Induced Hepatotoxicity,” Turkey Journal of Gastroenterology, Vol. 9, No. 3, 2009, pp. 204-206.

[8] Direct Healthcare Professional Communication Regarding Moxifloxacin (Avelox ${ }^{\circledR}$ ) and Serious Hepatic and Bullous Skin Reactions. Bayer HealthCare Medical Information Department, (Medicines and Healthcare Products Regulatory Agency (MHRA), Bayer). www.yeuowcard.gov.ukh 
[9] N. E. Henann and M. F. Zambie, "Gatifloxacin-Associated Acute Hepatitis,” Pharmacotherapy, Vol. 21, No. 12, 2001, pp. 1579-1582. doi:10.1592/phco.21.20.1579.34479

[10] B. S. Thakur, A. I. Jain, S. Sirkar, G. Joshi, and R. Joshi, "Ciprofloxacin-Induced Cholestatic Jaundice," Indian Journal of Gastroenterology, Vol. 26, 2007, pp. 51-52.

[11] A. Blum, "Ofloxacin-Induced Acute Severe Hepatitis," Southern Medical Journal, Vol. 84, 1991, p. 1158. doi:10.1097/00007611-199109000-00027

[12] L. Xiao and C. Sheng, "Anaphylactic Shock with Liver Damage Caused by Levofloxacin Injection," Chinese Journal of Drug Application and Monitoring, Vol. 7, No. 5, 2010, p. 32.

[13] FDA Issues Public Health Advisory on Liver Toxicity Associated with the Antibiotic Trovan [FDA Talk Paper], US Department of Health and Human Services, Food and Drug Administration, Rockville, 1999.

[14] D. N. Fish and D. S. North, "Gatifloxacin an Advanced 8-Methoxy Fluoroquinolone,” Pharmacotherapy, Vol. 21, No. 1, 2001, pp. 35-59. doi:10.1592/phco.21.1.35.34440

[15] T. Vial, M. Biour, J. Descotes and C. Trepo, “Antibiotics Associated Hepatitis Update from 1990," Annals of Pharmacotherapy, Vol. 31, 1997, pp. 204-220.

[16] O. Cheung, K. Chopra, T. Yu and A. Obaid, "Gatifloxacin Induced Hepatotoxicity," Annals of Internal Medicine, Vol. 140, 2004, pp. 72-73.

[17] C. I. Coleman, J. V. Spencer, J. O. Chung and P. Reddy, "Possible Gatifloxacin-Induced Fulminant Hepatic Failure,” Annals of Pharmacotherapy, Vol. 36, No. 78, 2002, pp. 1162-1167. doi:10.1345/aph.1A414

[18] K. Von Seggerm, R. Russo and M. A. Wikler, “A Novel Approach to Post Marketing Surveillance: The Tequin Clinical Experience Study [Abstr]," Program and Abstracts of the 40th Interscience Conference on Antimicrobial Agents and Chemotherapy, American Society for Microbiology, Washington DC, 2000, p. 468.

[19] E. V. Caparelli, M. D. Reed, J. S. Bradley, G. L. Kearns, R. F. Jacobs, B. D. Damle, J. L. Blumer and D. M. Grasela, "Pharmacokinetics of Gatifloxacin in Infants and Children,” Antimicrobial Agents Chemotherapy, Vol. 49, No. 3, 2005, pp. 1106-1112. doi:10.1128/AAC.49.3.1106-1112.2005

[20] S. E. Orman, H. S. Conjevaram, R. Vuppalachi, W. T. Freston, J. Rochen, E. D. Kleiner and P. H. Hayashi, "Clinical and Histopathologic Features of Fluoroquinolone- Induce Liver Injury,” Clinical Gastroenterology and Hepatology, Vol. 9, No. 6, 2011, pp. 517-523. doi:10.1016/j.cgh.2011.02.019

[21] A. Bogzil, G. Shams and S. Malhat, "Some Toxicological Heamatological and Biochemical Studies on Gatifloxacin in Rats," Global Journal of pharmacology, Vol. 4, No. 3, 2001, pp. 151-153.

[22] H. Liu, "Safety Profile of the Fluoroquinolones: Focus on Levofloxacin,” Drug Safety, Vol. 33, No. 5, 2001, pp. 353-369. doi:10.2165/11536360-000000000-00000

[23] S. Coban, B. Ceydilek, F. Ekiz, E. Erden and L. Soykan, "Levofloxacin-Induced Acute Fulminant Hepatic Failure in a Patient with Chronic Hepatitis B Infection,” Annals of Pharmacotherapy, Vol. 39, 2005, pp. 1737-1740. doi:10.1345/aph.1G111

[24] F. Carrascosa, M. I. Lucena, R. J. Andrade, J. S. Caviedes, A. C. Lavin, J. C. Mones, A. P. Vicente, B. Serrano and V. B. Serrano, "Fatal Acute Hepatitis after Sequential Treatment with Levofloxacin, Doxycycline, and Naproxen in a Patient Presenting with Acute Mycoplasma Pneumonia Infection,” Clinical Therapeutics Vol. 31, No. 5, 2009, pp. 1014-1019. doi:10.1016/j.clinthera.2009.05.012

[25] K. Airey and E. Koller, "Acute Hepatitis Associated with Levofloxacin in a Patient with Renal Insufficiency,” Canadian Medical Association Journal, Vol. 169, 2003, p. 8

[26] A. Karim, S. Ahmed, L. J. Rossoff, R. K. Siddiqui and H. N. Steinberg, "Possible Levofloxacin-Induced Acute Hepatocellular Injury in a Patient with Chronic Obstructive Lung Disease,” Clinical Infectious Disease, Vol. 33, No. 20, 2001, pp. 88-90.

[27] T. Papastavros, L. T. Dolovich, A. Holbrook, L. Whitehead and M. Loeb, "Adverse Events Associated with Pyrazinamide in the Treatment of Latent Multidrug Resistant Tuberculosis," Canadian Medical Association Journal, Vol. 167, No. 2, 2002, pp. 131-136.

[28] J. D. Schwalm and C. H. Lee, “Acute Hepatitis Associated with Oral Levofloxacin Therapy in a Hemodialysis Patient," Canadian Medical Association Journal, Vol. 168, 2003, pp. 847-848.

[29] L. Spahr, L. Rubbia Brandt, O. Marinescu, B. Armenian and A. Hadengue, "Acute Fatal Hepatitis Related to Levofloxacin,” Journal of Hepatology, Vol. 35, No. 3, 2001, pp. 8-9.

[30] D. Mennecier, C. Thiolet, C. Bredin, Potier, B. Vergeau and O. Farret, "Acute Pancreatitis after Treatment with Levofloxacin and Methylprednisolone,” Gastroenterologie Clinique Biologigue, Vol. 25, 2001, pp. 921-922.

[31] M. Hunt, "Levofloxacin: Dysglycemia and Liver Disorders,” Canadian Adverse Reaction Newsletter, Vol. 17, No. 1. 2007, pp. 1-2. www.healthcanada.gc.ca/carn

[32] Levoquin (Levofloxacin) Product Monogram, Jansen Inc., Toronto, Ontario, M3C IL9, Revised July 2011.

[33] J. B. Kahn, "Latest Industry Information on the Safety Profile of Levofloxacin in the US," Chemotherapy, Vol. 47, Suppl. 3, 2001, pp. 32-37. doi:10.1159/000057842

[34] K. Yagawa, "Latest Industry Information on the Safety Profile of Levofloxacin in Japan,” Chemotherapy, Vol. 47, No. 3, 2001, pp. 38-43. doi:10.1159/000057843

[35] A. Koverech, M. Picari, F. Granata, R. Fostini, D. Toniolo and G. Recchia, "Safety Profile of Ofloxacin," Italian Data Base Infection, Vol. 14, No. 4, 1986, pp. 335337.

[36] F. S. Jones and R. H. Smith, "Quinolones May Induce Hepatitis,” British Medical Journal, Vol. 314, 1997, p. 869. doi:10.1136/bmj.314.7084.869

[37] P. Gonzalez Carro, M. L. Huidobro, A. P. Zabala and E. M. Vicente, "Fatal Subfulminant Hepatic Failure with Ofloxacin,” American Journal of Gastroenterol, Vol. 95, No. 6, 2000, p. 1606.

doi:10.1016/S0002-9270(00)00910-2 
[38] M. Chaudhary, A. Tamta and R. Sehgal, "Sub-Chronic Toxicity Study of Fixed Dose Combination of OfloxacinOrnidazole in Musmusculus Mice,” The Open Toxicology Journal, Vol. 3, 2009, pp. 24-29.

[39] V. Onrust, M. Lamb, B. Barman and A. Julia. "Ofloxacin: A Reappraisal of Its Use in the Management of Genitourinary Tract Infections,” Drugs, Vol. 56, No. 5, 1998, pp. 895-928.

[40] M. I. Lucena, R. J. Andrade, H. S. Martinez, J. M. PerezSerrano and A. Gomez-Outes, "Norfloxacin-Induced Eosinophilic Necrotizing Granulomatous Hepatitis," American Journal of Gastroenterology, Vol. 95, No. 12, 2000, p. 3662.

[41] M. Romero-Gomez, E. S. Garcia, and M. C. Fernandez, "Norfloxacin-Induced Acute Cholestatic Hepatitis in a Patient with Alcoholic Liver Cirrhosis," American Journal of Gastroenterology, Vol. 94, 1999, pp. 2324-2325. doi:10.1111/j.1572-0241.1999.02324.X

[42] M. I. Lucena, R. J. Andrade, H. S. Martinez, J. M. Perez-Serrano and A. Gomez-Outes, "Norfloxacin Induced Cholestatic Jaundice,” American Journal of Gastroenterology, Vol. 93, No. 2, 1998, p. 2309. doi:10.1111/j.1572-0241.1998.02309.x

[43] A. G. Gilman, T. N. Rail, A. S. Nies and P. Taylor, "The Pharmacological Basis of Therapeutics,” 8 Edition, Pergamon, New York, 1990, pp. 1057-1060.

[44] Y. J. Drabo, A. Niakara and H. Ouedraogo, “Acute Pancreatitis Secondary to Administration or Norfloxacin," Annales Francasises d Anesthesie et de Reanimation, Vol. 21, 2002, pp. 68-69. doi:10.1016/S0750-7658(01)00562-7

[45] E. B. Bjomsson, R. Olsson and H. Remotti, "NorfloxacinInduced Eosinophilic Necrotizing Granulomatous Hepatitis,” American Journal of Gastroenterology, Vol. 95, No. 12, 2000, p. 3662.

[46] A. Maura, A. Dino, A. Gardella and C. Falugi, "Micronucleus Formation in Fetal Maternal Rat Erythroblasts after Norfloxacin Transplacental Administration,” Mutatio Research, Vol. 312, No. 2, 1994, pp. 127-130.

[47] Noroxin (Norfloxacin) Prescribing Information, K, MERCK and CO. INC White House Station, NJ.

[48] A. Torres, J. F. Muri, P. Corris, R. Kubin, I. Duprat-Lomon, P. P. Sagnier and G. Hoffken, "Effectiveness of Oral Moxifloxacin as Standard First Line Therapy in Community Acquired Pneumonia,” European respiratory Journal, Vol. 21, 2003, pp. 135-143. doi:10.1183/09031936.03.00045202

[49] R. Verma, R. Dhamija, D. Batts, S. C. Rossand and M. E. Loehrke, "Moxifloxacin Induced Fatal Hepatotoxicity in a 72-Year-Old Man: A Case Report," Cases Journal, Vol. 2, 2009, pp. 1-3. doi:10.4076/1757-1626-2-8063

[50] S. Nori, C. Nebesio, R. Brasheav and J. B. Travers, "Moxifloxacin Associated Drug Hypersensitivity Syndrome with Toxic Epidermal Necrolysis and Fulminant Hepatic Failure,” Archives of Dermatology, Vol. 104, 2004, pp. 1537-1538. doi:10.1001/archderm.140.12.1537

[51] S. Soto, L. Lopez-Roses, A. Avila Lancho, A. Gonzalez, E. Santos and B. Urraca, "Moxifloxacin-Induced Acute Liver Injury,” American Journal Gastroenterology, Vol.
97, 2002, pp.1853-1854. doi:10.1111/j.1572-0241.2002.05873.x

[52] Bayer Corporation, Avelox Safety Profile. http://www.avelox.com/Aveloxlavx_iv_safety.htm

[53] E. Von Kentz and G. Schluter, "Preclinical Safety Evaluation of Moxifloxacin a Novel Fluoroquinolone,” Journal of Antimicrobial Chemotherapy, Vol. 43, Suppl. B, 1999, pp. 91-100.

[54] P. B. Iannini, R. Kubin, C. Reiter and G. Tillotson, "Reassuring Safety Profile of Moxifloxacin,” Clinical Infectious Disease, Vol. 32, No. 1, 2001, pp. 1112-1114. doi:10.1086/319615

[55] C. Ho, Y. Chen, F. Hu, C. Yu, P. Yang and R. Lun, "Safety of Fluoroquinolone Use in Patients with Hepatotoxicity Induced by Anti-Tuberculosis Regimen,” Clinical Infections Disease, Vol. 48, 2009, pp. 1526-1533. doi:10.1086/598929

[56] P. Ball, L. Mandell, Y. Niki and G. Tillotson, “Comparative Tolerability of the Newer Fluoroquinolone Antibacterial,” Drug Safety, Vol. 2l, 2009, pp. 407-421.

[57] D. Hooper, “Quinolones,” In: G. L. Mandell, J. E. Bennett and R. Dolin, Eds., Mandell, Douglas, and Bennett's Principles and Practice of Infectious Diseases, 5th Edition, Churchill Livingstone, Philadelphia, 2000, pp. 404 423.

[58] F. Pea, F. Pavan and E. Lugatti, "Pharmacokinetic and Pharmacokinetic and Pharmacodynamics Aspect of oral Moxifloxacin $400 \mathrm{mg} 1$ Day in Elderly Patients with Acute Exaorbation of Chronic Bronchitis," Clinical Pharmacokinetics, Vol. 45, 2006, pp. 287-295. doi:10.2165/00003088-200645030-00004

[59] A. Zimpfer, A. Prospst, G. Mikuz, W. Vogel, L. Terracciano and S. Stadmann, "Ciprofloxacin Induced Acute Liver Injury: Case Report and Review of Literature,” Virchows Archive, Vol. 444, No. 1, 2004, pp. 87-89. doi:10.1007/s00428-003-0917-9

[60] J. K. Labowitz and W. B. Silverman, "Cholestatic Jaundice Induced by Ciprofloxacin,” Digestive Disease and Science, Vol. 42, 1997, pp. 192-194. doi:10.1023/A:1018870029216

[61] A. Aggarwal and J. Gurka, "Probable Ciprofloxacin Induced Cholestasis," Australian New Zealand Journal Medicine, Vol. 25, No. 5, 1995, pp. 541-542. doi:10.1111/j.1445-5994.1995.tb01506.x

[62] J. P. Villeneuve, C. Davies and J. Cole, "Suspected Ciprofloxacin-Induced Hepatotoxicity," Annals of Pharmacotherapy, Vol. 29, 1995, pp. 294-296.

[63] A. C. Hirsch and L. M. Lundquist, "Ciprofloxacin-Induce Hepatotoxicity Resolved with Levofloxacin: A Case Report and a Review of the Literature," Hospital Pharmacy, Vol. 44, No. 11, 2009, pp. 978-983. doi:10.1310/hpj4411-978

[64] A. J. Dichiara, Alkinson, Z. Goodman and H. E. Sherman, "Ciprofloxacin-Induced Acute Cholestatic Liver Injury and Associated Renal Failure Case Report and Review," Minerva Gastroenterologica e Dietologica, Vol. 54, No. 3, 2008, pp. 307-315.

[65] Z. M. Goetz, P. R. Galle and A. Schwarting, "Non Fatal 
Acute Liver Injury Possibly Related to High-Dose Ciprofloxacin," European Journal of Clinical Microbiology and Infectious Disease, Vol. 22, No. 5, 2003, pp. 294296.

[66] M. Alcalde, M. S. Donoso, M. Carda Diaz and J. M. Puscosio Narvaezi, "Liver Dysfunction Due to Ciprofloxacin,” Acta Gastroenterologica Belgica, Vol. 58, No. 5, 1995, pp. 475-476.

[67] O. Sherman and J. L. Beizer, "Possible CiprofloxacinInduce Acute Cholestatic Jaundice,” Annals of Pharmacotherapy, Vol. 28, No. 10, 1994, pp. 1162-1164.

[68] E. Cholongitas, C. Georgousaki, S. Spyrou and M. Deisenaki, "Ciprofloxacin Induce Acute Cholestatic Hepatic Jaundice,” Annals of Hepatology, Vol. 83, No. 4, 2001, pp. 400-401.

[69] M. Pfeiffer, C. Reiter, S. Fuchs and M. Simonz, "Fatal Hepatic Failure Associated with Ciprofloxacin,” Lancet, Vol. 343, No. 8899, 1994, pp. 738-739. doi:10.1016/S0140-6736(94)91624-1

[70] B. K. Grassmick, V. T. Lehr and A. S. Sundareson, "Fulminant Hepatic Failure Possibly Related to Ciprofloxacin,” Annals of Pharmacotherapy, Vol. 26, No. 5, 1992, pp. 636-639.

[71] S. A. Zaidi, "Hepatitis Associated with Amoxicillin/ Clavulanic Acid and/or Ciprofloxacin,” American Journal of Medical Sciences, Vol. 325, 2003, pp. 31-33. doi:10.1097/00000441-200301000-00006

[72] S. Fuchs, Z. Simeon and M. Brezis, "Fatal Hepatic Failure Associated with Ciprofloxacin,” Lancet, Vol. 19, No. 343, 1994, pp. 738-739. doi:10.1016/S0140-6736(94)91624-1

[73] J. R. Levinson and A. Kumar, "Ciprofloxacin-Induced Cholestatic Jaundice: A Case Report (Abstract),” American Journal of Gastroenterology, Vol. 88, 1993, p. 1619.

[74] M. A. Channa and M. Z. Janjua, "Effects of Ciprofloxacin on Fetal Hepatocytes," Journal of Pakistan Medical Association, Vol. 53, No. 10, 2003, pp. 448-450.

[75] G. Y. Minuk, N. Assy and L. X. Ding, "Effects of Quinolone Antibiotic on Hepatic Growth and Protein Synthesis Following Partial Hepatectomy in Rats," Journal of Gastroenterology and Hepatology, Vol. 12, 1997, pp. 5-7. doi:10.1111/j.1440-1746.1997.tb00346.x

[76] A. Basaran, K. Erol, N. Basaran, H. V. Gunes, E. Acikalin, P. G. Timural, I. Degirmenci, E. A. Cakmak and A. G. Tomati, "Effect of Ciprofloxacin on Chromosomes, and Hepatic and Renal Functions in Rats," Chemotherapy, Vol. 39, No. 3, 1993, pp. 182-188. doi:10.1159/000239124

[77] H. I. Nadia, “Assessment of Histopathological and Histological Changes in Liver of Pregnant Female Rats then Fetuses Following Ciprofloxacin Administration," Journal of Egypt Society of Toxicology, Vol. 35, 2006, pp. 717.

[78] A. I. Weyers, U. I. Laura, G. O. Hugo and B. G. Nora, "Ciprofloxacin Increases Hepatic and Renal Lipid Hydroperoxide Levels in Mice,” Biocell, Vol. 26, No. 2, 2002, pp. 225-228.

[79] G. Y. Minuk, T. Gauthier, X. K. Zhang, G. Q. Wang and
F. T. Burczynski, "Ciprofloxacin Reverses the Inhibitory Effects of Acute Ethanol Exposure on Hepatic Regeneration in the Rat," Hepatology, Vol. 22, 1995, pp. 17971800.

[80] R. Stahlmann, "Clinical Toxicological Aspects of Fluoroquinolones,” Toxicology Letters, Vol. 127, 2002, pp. 269277. doi:10.1016/S0378-4274(01)00509-4

[81] H. J. Chen, K. J. Bloch and J. A. Maclean, “Acute Eosinophilic Hepatitis from Travofloxacin,” New England Journal of Medicine, Vol. 42, No. 5, 2000, pp. 359-360. doi:10.1056/NEJM200002033420517

[82] D. A. Lazarczyk, N. S. Goldstein and S. C. Gordon, “Abstract Travofloxacin Hepatotoxicity,” Digestive Disease and Sciences, Vol. 46, No. 4, 2001, pp. 925-926. doi:10.1023/A:1010741510046

[83] K. H. Pannu, L. Gohlieb and K. E. Fishman, “Acute Liver Failure Due to Travofloxacin; CT Findings,” Emergency Radiology, Vol. 8, 2001, pp. 108-110. doi:10.1007/PL00011876

[84] J. Borlak, “Travofloxacin: A Case Study of Idiosyncratic or Iatrogenic Liver Toxicity-Molecular Mechanism and Lessons for Pharmacotoxicity," Human and Experimental Toxicology, Vol. 28, 2004, pp. 119-121. doi:10.1177/0960327109105767

[85] M. I. Lucena, R. J. Andrade, L. Rodrigo, J. Salmeron, A. Alvarez, M. J. Lopez-Garrido, R. Camargo and R. Acantara, "Travofloxacin Induced Acute Hepatitis," Clinical Infectious Disease, Vol. 30, No. 2, 2000, pp. 400-401. doi:10.1086/313680

[86] M. J. Liguori, M. G. Anderson, S. Bukofzer, J. McKim and J. F. Waring, "Suggested Mechanism for Hepatotoxicity Induced by Travofloxacin,” Hepatology, Vol. 4, No. l, 2005, pp. 177-186. doi:10.1002/hep.20514

[87] FDA Medical Officer's Safety Update, April-June 1998, pp. 5-6.

[88] FDA Pharmacology Review, 18 December 1997.

[89] Trovanproject Monogram Produced by Pfizer Inc., 1998, Reviewed July 1998.

[90] Travofloxacin/Alatrofloxacin, Pharmacology Review, 1997, pp. 43-44.

[91] J. F. Waring, M. J. Liguori, J. P. Luyendyk, J. F. Maddox, P. E. Ganey, R. F. Stachlewitz, C. North, E. A. Blomme and R. A. Roth, "Microarray Analysis of Lipopolysaccharide Potentiation of Travofloxacin Induced Liver Injury in Rats Suggests a Role for Proinflammatory Chemokines and Neutrophils," Journal of Pharmacology and Experimental Therapeutics, Vol. 316, No. 3, 2006, pp. 10801087. doi:10.1124/jpet.105.096347

[92] P. J. Shaw, M. I. Hopfensperger, P. E. Ganey and R. A. Roth, "Lipopolysaccharide and Travofloxacin Coexposure in Mice Causes Idiosyncrasy Like Liver Injury Dependent on Tumor Necrosis Factor-Alpha,” Toxicological Sciences, Vol. 100, No. 1, 2007, pp. 259-266. doi:10.1093/toxsci/kfm218

[93] Travofloxacin/Alatrofloxacin, Pharmacology Review, 18 December 1997, p. 42.

[94] Public Statement on Trovan/Trovan IV/Turvel/Turvel IV (Travofloxacin/Alatrofloxacin) [Press Release 15770/99]. 
European Agency for the Evaluation of Medicinal Products, London, 25 May 1999.

[95] G. Labbe, D. Pessayre and B. Fromenty, "Drug Induced Liver Injury through Mitochondria Dysfunction; Mechanisms and Detection during Preclinical Safety Studies,” Fundamental and Clinical Pharmacology, Vol. 22, 2008, pp. 335-353. doi:10.1111/j.1472-8206.2008.00608.x

[96] M. P. Holt and C. Ju, "Mechanism of Drug Induced Liver Injury," American Association of Pharmaceutical Scientists Journal, Vol. 8, No. 1, 2006, pp. 48-54.

[97] C. J. Hsiao, H. Younis and U. A. Boelsterli, “Travofloxacin, a Fluoroquinolone Antibiotic with Hepatotoxic Potential, Causes Mitochondrial Peroxynitrite Stress in a Mouse Model of Underlying Mitochondrial Dysfunction," Chemico-Biological Interactions, Vol. 188, No. 1, 2010, pp. 204-213. doi:10.1016/j.cbi.2010.07.017

[98] Q. Sun, R. Zhu, F. W. Foss and T. L. Macdonald, “In Vitro Metabolism of a Model Cyclopropylamine to Reactive Intermediate: Insights into Travofloxacin-Induced Hepatotoxicity," Chemical Research in Toxicology, Vol. 21, No. 3, 2008, pp. 711-719. doi:10.1021/tx7003085
[99] P, Jones, J. Lemasters, D. Han, A. U. Boelsterli and N. Kaplowitz, "Mechanisms of Pathogenesis in Drug Hepatotoxicity, Putting the Stress on Mitochondria," Molecular Interventions, Vol. 10, No. 2, 2010, pp. 98-111. doi:10.1124/mi.10.2.7

[100] A. A. Pino, A. V. Maura and L. Masciangelo, "Evaluation of DNA Damage Induced by Norfloxacin in Liver and Kidney of Adult Rats and Fetal Tissues after Transplacental Exposure,” Mutation Research, Vol. 264, 1991, pp. 81-85. doi:10.1016/0165-7992(91)90049-A

[101] Y. Kashida, Y. Sasaki, K. Oshawa, N. Yokohama, A Takahashi, T. Watanabe and K. Mitsumori, "Mechanistic Study on Flumequine Hepatocarcinogenicity Focusing on DNA Damage in Mice,” Toxicological Science, Vol. 69, No. 2, 2002, pp. 317-321. doi:10.1093/toxsci/69.2.317

[102] F. Hincal and T. Taskin, "The Mechanism Induced by Ciprofloxacin May Involve the Generation of Free Radicals and the Activation of Excitatory Amino Acid Receptors," Abstracts of the International Congress of Toxicology_VII Seattle, W.A. Abstr, No. 99, 1995, p. 27. 